\title{
Heat Transfer and Fluid Flows in Gas Springs
}

\author{
U. Lekic ${ }^{*}$ and J.B.W. Kok
}

University of Twente, Enschede, The Netherlands

\begin{abstract}
Thermodynamical processes and fluid mechanics phenomena occurring in an unlubricated piston-cylinder gas spring are investigated. Existing instantaneous heat transfer correlations are not applicable to the piston-cylinder compressed volumes. Accurate prediction of the transient thermal properties, heat transfer and fluid flows for the high performance, tight tolerance and lubricant-free gas compressors (e.g. cryo-cooling industry) is of high industrial importance.

Research is here done with the use of ANSYS CFX finite-volume numerical package, modelling the closed fluid domain in a valveless non-lubricated one-piston-cylinder gas spring. Obtained numerically simulated values are compared to experimentally measured pressure and heat-flux data; very good agreement is observed. Broad discussion and conclusions with regard to thermodynamic and fluid-flow phenomena occurring are presented.
\end{abstract}

Keywords: Thermodynamics in gas springs, CFD modelling, heat transfer and fluid flows.

\section{INTRODUCTION}

Piston-cylinder based machinery is in use in a very broad range of industrial applications. Examples are internal combustion engines in the automotive, marine and aerospace applications, compressors in the cooling devices for domestic and commercial applications, for the gas liquefaction, medical purposes and superconductivity technology in electronics. All of them are characterized by the moving pistons inside cylindrical volumes compressing the enclosed operating fluid. These parts are conventionally lubricated to reduce the frictional forces and wear, and cool the contact surfaces. Unfortunately, lubricants inevitably pollute the working medium. In the open-cycle internal combustion engines this is acceptable to reasonable limits (the lubricant vapour or droplets can be combusted and removed), but when it comes to closed cooling-cycle systems, any presence of impurities in the thermal carrier is highly undesirable, and efforts are put into avoiding it. Ways to achieve this are thorough filtering of the operating medium, careful sealing, and shortening of the maintenance intervals. The other direction, leading the course of the project the work presented here is part of, is to omit the lubrication itself, and thus eliminate the related decrease of operational performance and increase in the constructional and consequential operating costs.

Accurate thermal analyses are crucial in this line of considerations. Thermal expansion, internal stresses and distortion of the projected geometric relations can lead to adverse frictional stresses, delamination and destruction of the exposed and interacting parts, wear and fatigue. The oilfree constructions investigated here demand extremely narrow tolerances of the functional parts $(\leq 20 \mu \mathrm{m})$, which

*Address correspondence to this author at the University of Twente, Enschede, The Netherlands; Tel: +31534893661 ; Fax: +31534892507 ; E-mail: u.lekic@utwente.nl and description of the heat transfer phenomena occurring between the gas and enclosing solid parts. Processes occurring in a piston gas spring represent qualitatively the thermodynamic processes occurring in reciprocating piston compressors, eliminating the effects of inflow and discharge streams. For these reasons, here presented research concentrates on better understanding and improved modelling of the heat transfer mechanisms and fluid-flow phenomena occurring in piston gas springs.

In the past century extensive work was done and published on the understanding of steady-state convective heat transfer. This resulted in the Nusselt-Reynolds $(\mathrm{Nu}-\mathrm{Re})$ heattransfer analogy and all its successor relations, boundary layer theory, and the turbulence closure models for the nearwall region fluid flows. Generally accepted conventional $\mathrm{Nu}$ Re correlations were derived for the steady state developed boundary layer flows, and are generally applicable to a number of specific fluid-flow types (in-pipe, around the cylinder, over the flat plate), and can be expanded to a certain extent to the similarly characterized flows. Nevertheless these are not applicable to the transient heat transfer from rapidly compressed and expanded gas to the surrounding walls. In piston-cylinder constructions, phase shift has been observed between the peak instantaneous heat flux at the gas spring walls and the peak bulk gas temperature.

The peak heat flux precedes the temperature-difference maximum up to as much as $45^{\circ}$ Crank-Angle (CA), (Fig. 1 [1-3]). Attempts were made and have been reported in the literature $[1,3,4]$ to include this phenomenon in the conventional $\mathrm{Nu}-\mathrm{Re}$ model by adding a complex phasing part to the bulk gas temperature - heat transfer correlation. This, however, has not led to a generically applicable correlation, but to a series of geometry- and operating conditions-specific expressions. 


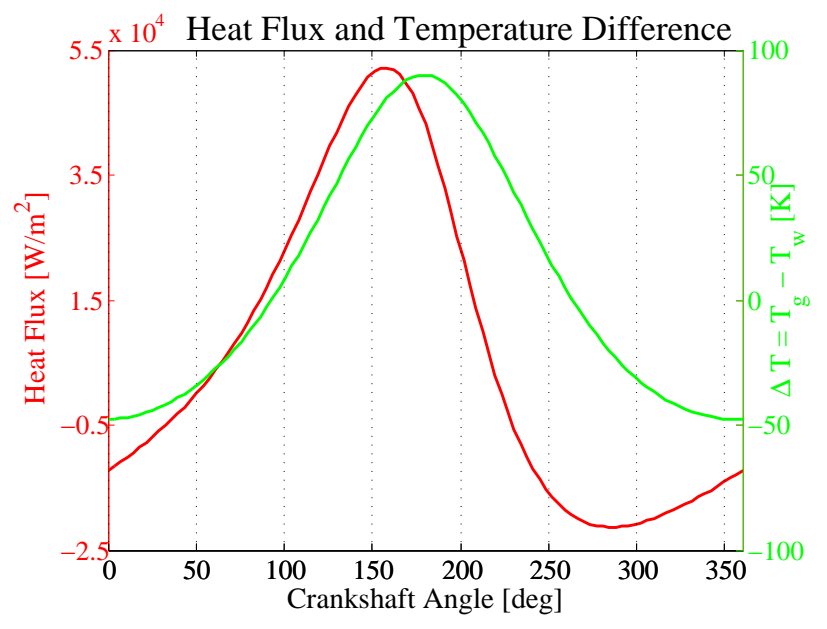

Fig. (1). Temperature and heat flux phase shift.

In the presented work, a numerical approach to this problem is explored. Heat transfer between the compressed gas and the surrounding walls is modelled with Computational Fluid Dynamics (CFD), applied on the flow in a closed domain with transiently changing volume. Commercially available, finite-volume code ANSYS CFX11.0 is used for the numerical discretization and calculation of the gas flow field, wall heat flux and heat conduction in the solid wall. Underlying governing equations are the standard unsteady compressible Navier-Stokes equations set up in ANSYS CFX [5]. A Direct Numerical Simulation (DNS) approach is adopted; no approximating turbulence models are being used.

\section{METHODOLOGY}

Presented numerical work is formatted to, and validated on the data generated at Massachusetts Institute of Technology (MIT) as part of a PhD project. Measurements have been performed on the experimental setup built and operated by professor A.A.Kornhauser, who made the measurement database and his doctoral thesis available to the authors. MIT gas spring setup description, together with the instrumentation specifics, was reported in [3]. Geometry of the numeri-

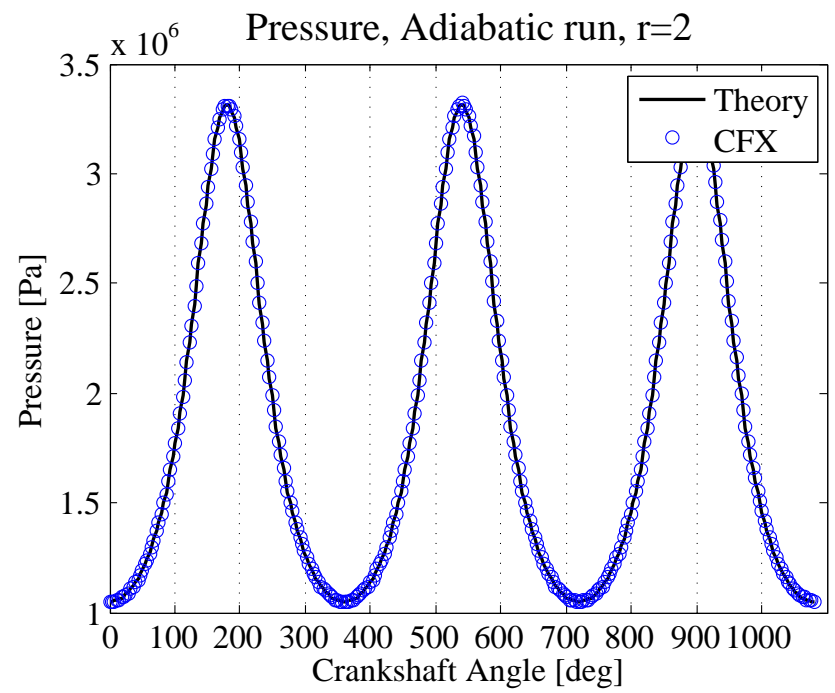

cal model, appropriate boundary conditions and initial conditions for simulations are taken according to experiments, to which the simulation results are compared to in the following chapters.

Using ANSYS Design Modeller, geometry design module in ANSYS, three-dimensional models are created according to the dimensions of the two base-line case MIT setups: two gas-springs with compression ratios 2 and 8, with constant diameter $D=50.8 \mathrm{~mm}$ and stroke $S=76.2 \mathrm{~mm}$; volume of the compressed gas is then varied with the height of the For reason of reduction in the computational time, and having in mind the fact that these gas springs are axi-symmetric, only $5^{\circ}$ cuts of the compressed volumes are modelled. Virtual geometries are then spatially discretized using CFX Mesh to a resolution sufficient to provide grid-independent numerical solutions. In this way created finite volume model is then exported to the CFX Pre module, where the solution parameterization is specified: the initial and boundary conditions for the computational model, temporal discretization parameters and selection of the relevant physical models to be numerically solved. Task is then forwarded to CFX Solver where the actual mathematical operations take place and the solutions are provided. Results are visualized using CFX Post.

As mentioned above, numerical models need to be adequately, both spatially and temporally, discretized to provide grid-independent solutions. As the general criterion, mass and energy conservation during the gas compression process and the mesh deformation are verified on a model with adiabatic walls and results are validated with the adiabatic thermodynamic analogy ( $p V^{\kappa}=$ const, $\kappa$ being the adiabatic exponent) over a period of 10 operating cycles (Fig. 2). Please note that $r$ in the figure caption denotes the compression ratio. Subsequently, models are generated also for nonadiabatic cases, as these are in the central scope of this research. Two operating frequencies, as the base-line cases in Kornhauser's work are investigated here: 2 RPM referred to as the slow run, and 1000 RPM as the fast run. Properties of interest, taken as directly obtainable results from the simulations, are the volume-averaged gas pressure and the surfaceaveraged instantaneous wall heat flux along the compression cycle. Finally, these are compared to the available experimental database; also fluid flow dynamics of the system are

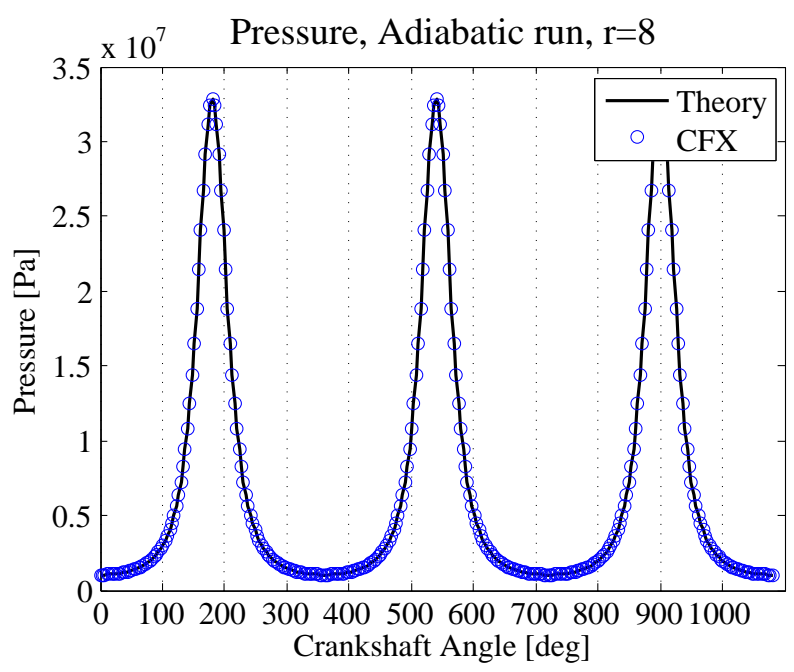

Fig. (2). Adiabatic run - numerical simulation vs. thermodynamic analogy for $r=2$ (left) and r=8 (right). 
examined and their influence to the heat transfer is discussed. Overview of the investigated dimensions and the operating conditions is given in Table 1. Dead space height in the table denotes the distance from the piston top to the flat cylinder top at the Top Dead Centre (TDC) piston position.

For all simulations, as mentioned in the introduction, a DNS approach is adopted. In previous work, [6], authors explored k- $\omega, S A S$ - Scale Adaptive Simulation and LES Large Eddy Simulation models, but this was abandoned for the apparent absence of actual developed turbulence in the compressed fluid, and the capability of the numerical code to solve the fluid flows without the use of approximating near-wall models. This is also discussed in the following chapters.

\section{NUMERICAL MODELLING}

As stated in Table 1, the diameter and stroke of the piston in the experimental machine for both compression ratios is $D x S=50.2 \times 76 \mathrm{~mm}$, operating on 2 and 1000 RPM. Simulation setup, meshing specifics, initial and boundary condi- tions, and finally the results of the simulations, are presented in the following pages.

\section{Geometry and Mesh}

The CFX-model geometry and mesh are dimensioned according to the MIT experimental setup (Figs. 3, 4); as mentioned, only a $5^{\circ}$ cut of the compressed domain is modelled. CFX Mesh application is used for meshing the domain; the mesh is structured, and generated using the Advancing Front and Extruded 2D Mesh meshing strategy. After several consecutive spatial refinements, the finite volume edge length was limited to $2 \mathrm{~mm}$, and the compression ratio 2 model is optimized to $\sim 28000$ nodes ( 20000 elements), with narrower layers close to the wall (Fig. 5). Spatial refinement was done to ensure the grid-independent solutions, as well as to provide sufficient resolution both near the wall and in the bulk volume, for the purposes of the qualitative fluid-flow visualisation and quantitative investigation of the radial temperature profiles and wall heat flux. ANSYS CFX Solver proved to be very robust with respect to the spatial resolution, and gave very accurate results for the pressure amplitude, even for a comparatively very course

Table 1. Base-Line Experimental Setup Dimensions and Operating Frequencies

\begin{tabular}{|c|c|c|c|}
\hline Model Dimensions \& Frequencies & Diameter [mm] & Stroke [mm] & Dead Space Height [mm] \\
\hline Compression ratio 2 & 50.8 & 76.2 & 76.2 \\
\hline Compression ratio 8 & 50.8 & 76.2 & 10.89 \\
\hline
\end{tabular}

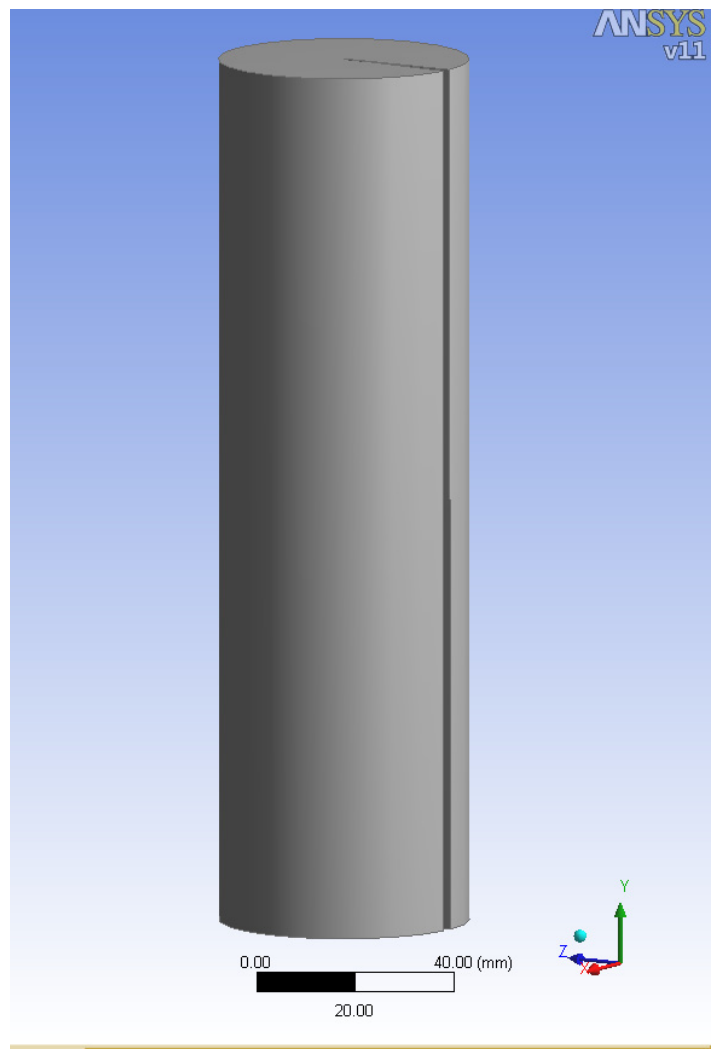

Fig. (3). The compression-ratio 2 gas spring model and the $5^{\circ}$ axial cut

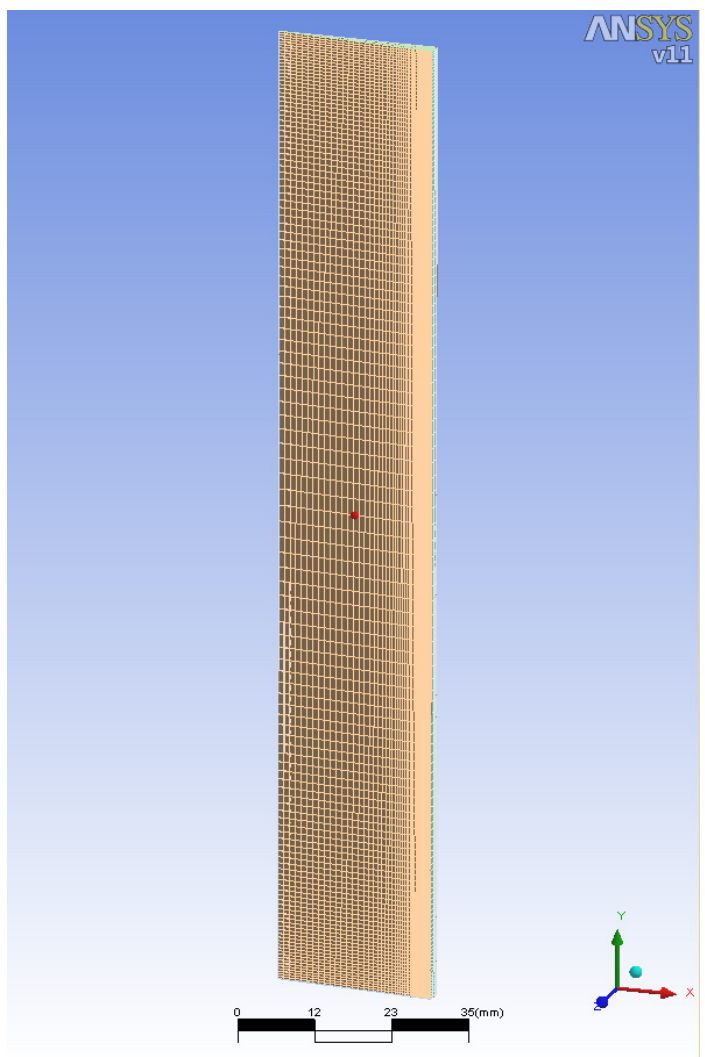

Fig. (4). Meshed 3D geometry of the gas spring model 

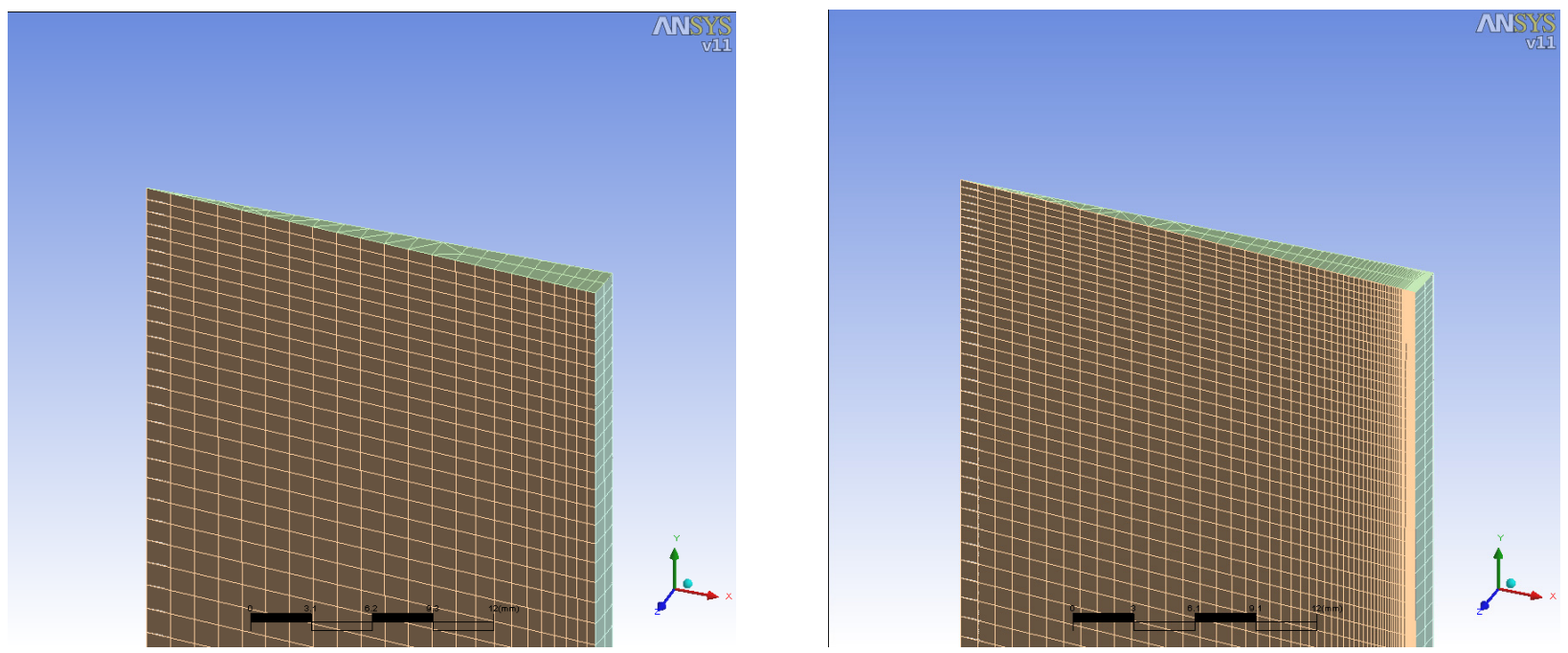

Fig. (5). Top-corner view of the domain for 2 different mesh resolutions: model with 6000 (left) and 28000 nodes (right figure).

mesh. Fig. (6) shows the pressure curve along the full cycle for three steps of mesh refinement: models with $\sim 6000$, $\sim 16000$ and $\sim 28000$ nodes; the error in peak pressure results for the coarsest mesh is no more than $1 \%$ of the cycle amplitude, while the other two give identical results. Wall heat flux results are more affected by the resolution at the wall, and the error for the coarsest mesh is in parts of the cycle over $100 \%$ (not shown here); while for the two other, finer meshes, heat flux results are also basically identical. Mesh with $\sim 16000$ nodes would thus give accurate results too; nevertheless mesh was refined for the flow visualization purposes, and the model displayed in Fig. (4), with 28000 nodes is used as the generic mesh for the compression ratio 2 simulations. Identical geometry and mesh are used for both slow and fast runs (mesh is optimized on the critical, fast run). Meshing parameters are then extrapolated and applied on the compression ratio 8 simulation models too.

\section{Boundary Conditions}

The model displayed on Fig. (3) and Fig. (4) represents the fluid - compressed gas domain. The five boundaries defining the computational domain (Fig. 4) are surfaces representing:

- the co-axial cylinder on the outer boundary,

- horizontal cylinder head,

- horizontal piston top, and

- two symmetry planes sharing the symmetry axis.

All the solid boundaries (cylinder, cylinder head and the piston) are prescribed in the code as wall boundaries, allowing no mass or momentum flux in the perpendicular direction. They are also imposed a no-slip condition, leading to the zero-velocity condition at the wall. The piston top is the only moving boundary in the model; the other boundaries are set as stationary in space. ANSYS CFX 11.0 allows for the moving/deforming mesh, and the mesh elements are allowed to compress and redistribute in the axial direction; every node displacement is specified through an explicit function in order to ensure uniform cell-distortion and avoid the occurrence of negative-volume elements. The piston displace- ment function is determined by the machine geometry, i.e. by Equation 1:

PistDisp $=S \cdot\left(a(1+\cos \theta)+\left(l-\sqrt{l^{2}-a^{2} \sin ^{2} \theta}\right)\right.$,

where PistDisp is piston displacement measured from the bottom-dead-centre (BDC), $S$ is the piston stroke, $a$ is the crankshaft throw, $l$ is the connecting rod length and $\theta$ is the crank angle also measured from BDC.

The piston surface (moving boundary) is set as an adiabatic wall. This reflects the situation in the test rig where the piston was not a cooled surface. The cylinder wall and the cylinder top are defined to be walls with a constant temperature of $295 \mathrm{~K}$, equal to the temperature of the coolant on the other side of the gas-spring walls. This approximation of the constant temperature was made in accordance to the fact that a solid cylinder liner has a very high thermal inertia with respect to the transient changes in the gas temperature, this resulting in very small actual oscillations in the wall surface temperature. This was measured for motored piston engines by several researchers, [7] and [1], and oscillation was shown to be no more than $\pm 3 \mathrm{~K}$ in amplitude, which is very small compared to the amplitude in the gas temperature. Validity of this assumption was also examined by [8], and here too it was shown that the wall temperature can be regarded as constant with a view to the effect on the wall heat flux. Symmetry planes are specified as Rotational Periodicity Interfaces, modelling the multiplication of the $5^{\circ}$ geometry around the symmetry axis and allowing for the mass, momentum and energy continuum in the azimuthal direction.

\section{Initial Conditions}

Compressed gas in the simulations was helium, as in the experiments from MIT. Kornhauser's measurements were recorded once the gas spring has reached a cyclic steady state in its operation, i.e. when the mean cycle pressure remained constant (less than 1\% deviation). Since data were available [3] only for compression ratio 2 and the two stated operating frequencies, the initial conditions for the simulations were different for these two particular cases, and as taken from these steady-state measurements. These are pre- 

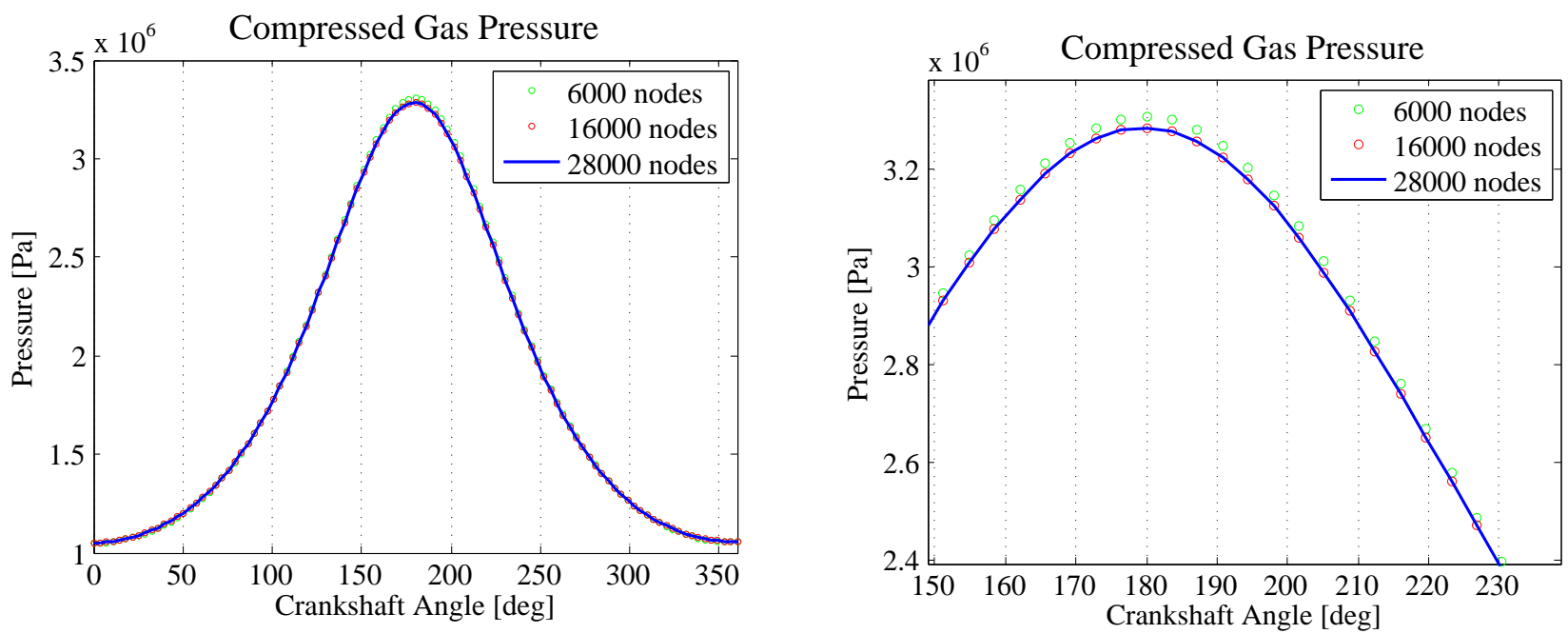

Fig. (6). Compared simulation results for different meshing resolutions.

sented in Table 2. Simulation runs were started from the BDC position, and all initial gas velocity components were set to zero. This is not in exact accordance with the experiments, but sufficient operating cycles were simulated in order to reach a numerical steady state and only then results were taken as representative.

Table 2. Initial Conditions for Simulations

\begin{tabular}{|c|c|c|}
\hline Initial Conditions & Pressure $[\mathbf{P a}] \cdot \mathbf{1 0}^{\mathbf{5}}$ & Temperature $[\mathbf{K}]$ \\
\hline Slow runs & 0.77757 & 295.3 \\
\hline Fast runs & 10.4859 & 245.4 \\
\hline
\end{tabular}

\section{Temporal Discretization}

The simulation model was also tested on the sensitivity to temporal discretization. ANSYS CFX uses a fully implicit solver, allowing for no restriction on the maximum Courant number for convergence. Thus the course of the temporal refinement wasn't taken with a view to the Courant number values, but in accordance with the recommendations in [5], and consecutively increasing the number of timesteps per cycle by a sensible factor. Results for 100, 500, 1000, 2000 and 4000 timesteps/cycle are shown in Fig. (7). There is a weak influence of the timestep size on the computed values, amounting to a maximum of $1.7 \%$ error of the pressure amplitude, as compared between the two extreme resolutions: 100 and 4000 timesteps/cycle. In the same purview, smaller time-steps naturally cause an increase in the computational costs. Having in mind negligible change with refinement after 2000 timesteps/cycle, and with acceptable computational costs, 2000 timesteps per cycle was the chosen cycle temporal resolution for all following simulations.

\section{Results}

As the full Navier-Stokes equations are being numerically solved, a large number of physical properties are directly producible and readable from the simulation results. In the scope of this paper, thermodynamical values of immediate interest are mass conservation throughout the cycle, and instantaneous values of gas pressure, gas temperature and the heat flux from the gas to the surrounding walls.

Pressure propagates by the speed of sound and therefore, having in mind the time-scales of the volume and density change, pressure could be assumed homogeneous in the compressed volume at every instance of the cycle. Due to the non-adiabatic, fixed-temperature walls as the gas boundaries, this could not be valid for the temperature of the gas; it
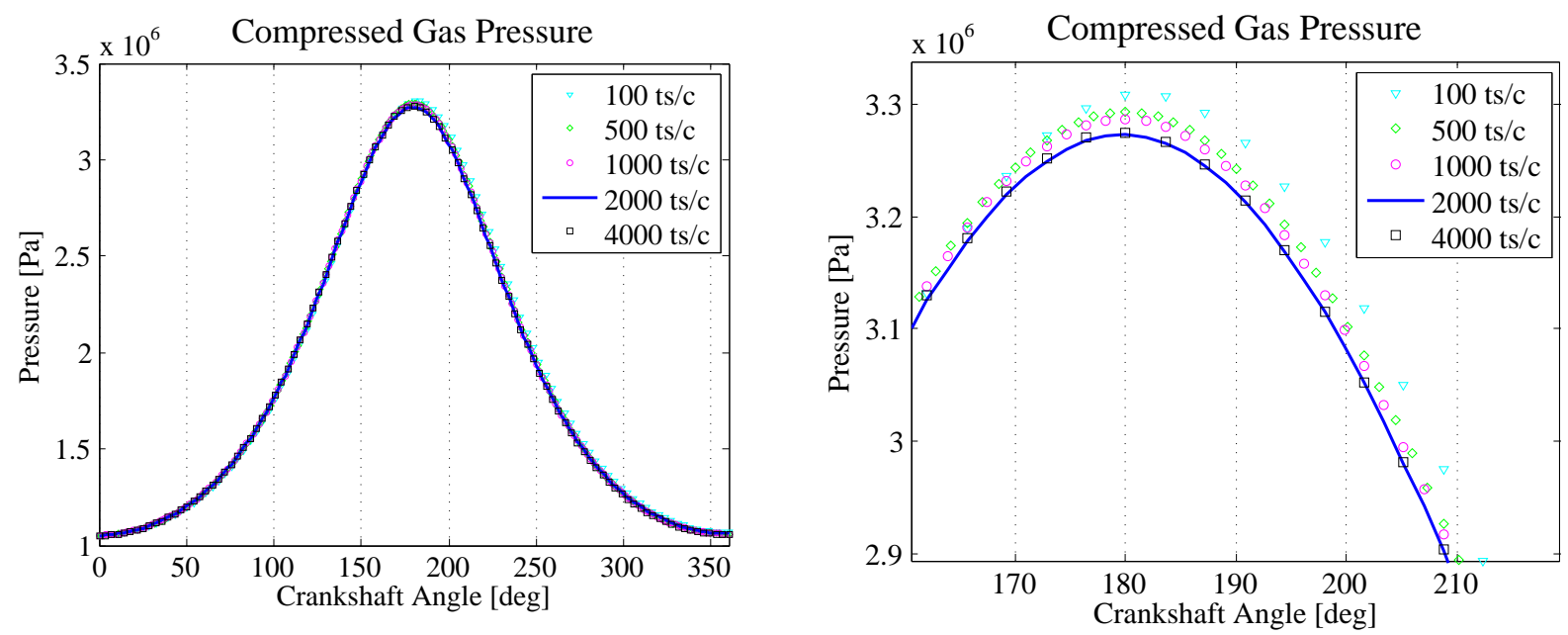

Fig. (7). Effects of temporal refinement on the pressure results. 
would vary significantly in both axial and radial directions causing the density gradients in the volume. Thus, the gas temperature presented here is the volume average of the density-weighted temperature of every discrete finite volume. In this way, this value approaches the theoretically uniform gas temperature stemming from the ideal-gas equation of state $(p V=m R T)$. Finally, the wall heat flux is averaged over the heat transfer surfaces - cylinder and the cylinder head. Mass of the gas is not shown here and is fully conserved throughout the cycle.

Results of the simulations are presented in Table $\mathbf{3}$ and Fig. (8) to Fig. (10). Table 3 shows the extreme values of gas pressure, temperature and wall heat flux, while Fig. (8) to Fig. (10) display the properties estimated against the immediate crank-angle position along one operating cycle, thus angle varying from $0^{\circ}$ (BDC) to $360^{\circ}, 180^{\circ}$ being the TDC. Peak values and amplitudes between the slow and fast runs cannot be directly quantitatively compared here due to different initial conditions, but the influence of the volumecompression ratio can be qualitatively examined. Amplitudes are naturally much larger for the higher compression ratio, especially for the faster runs where the processes approach adiabatic compression (as will be shown later), and the pressure changes in proportion to $\left(V / V_{\text {init }}\right)^{\kappa}$. Factor by which the maximum pressure at the compression end at TDC increases with the operating frequency and compression ratio ranges from $2 \times p_{\text {init }}$ for $r=2$ and the slow run, to $\sim 30 \times p_{\text {init }}$ for $r=2$ and the fast run, gas temperature reaching $\sim 900 \mathrm{~K}$ for the latter case. Wall heat flux ranges from $\sim 100 \mathrm{~W} / \mathrm{m}^{2}$, to as much as $\sim 750 \mathrm{~kW} / \mathrm{m}^{2}$. Work lost during one cycle, i.e. the gas energy dissipated by virtue of heat transfer to the surrounding solid, integrated over one compression cycle, grows from $0.35 \mathrm{~J}$ for slow operation of the $r=2$ gas spring to $\sim 20 \mathrm{~J}$ for the higher compression ratio and operating frequency.

Compression ratio 2 simulations will be validated on and compared to experimentally obtained results, while the results for compression ratio 8 are presented here for qualitative comparison. Thermodynamic and fluid flow considerations and conclusions made for $r=2$ can be extrapolated and are generally applicable to higher ratios as well.

\section{COMPARISON TO THE EXPERIMENTAL RESULTS}

\section{Experimental Setup}

The MIT experimental setup is a single piston-cylinder crankshaft driven gas spring without suction/exhaust valves. Cylinder liner and the cylinder top were made of steel, and piston was made of brass, sealed to the cylinder with a buna$\mathrm{n}$ o-ring to reduce leakage past the piston from the compressed space. Kornhauser experimented with a range of

Table 3. Results of the Simulations

\begin{tabular}{|c|c|c|c|c|}
\hline Initial Conditions & Pressure $[\mathrm{Pa}] \cdot 10^{5}$ & Temperature [K] & & \\
\hline Max values $r=2$ & Pressure $[\mathrm{Pa}] \cdot 10^{5}$ & Temperature [K] & Wall Heat Flux $\left[\mathrm{W} / \mathrm{m}^{2}\right]$ & Cyclic Work Loss [J] \\
\hline Slow runs & 1.5608 & 299.9 & 108.93 & 0.3510 \\
\hline Max values $r=8$ & Pressure $[\mathrm{Pa}] \cdot 10^{5}$ & Temperature [K] & Wall Heat Flux $\left[W / m^{2}\right]$ & Cyclic Work Loss $[\mathbf{J}]$ \\
\hline Slow runs & 6.4450 & 322.2 & 873.52 & 3.2720 \\
\hline Fast runs & 316.7920 & 928.7 & 757945 & 19.4283 \\
\hline
\end{tabular}
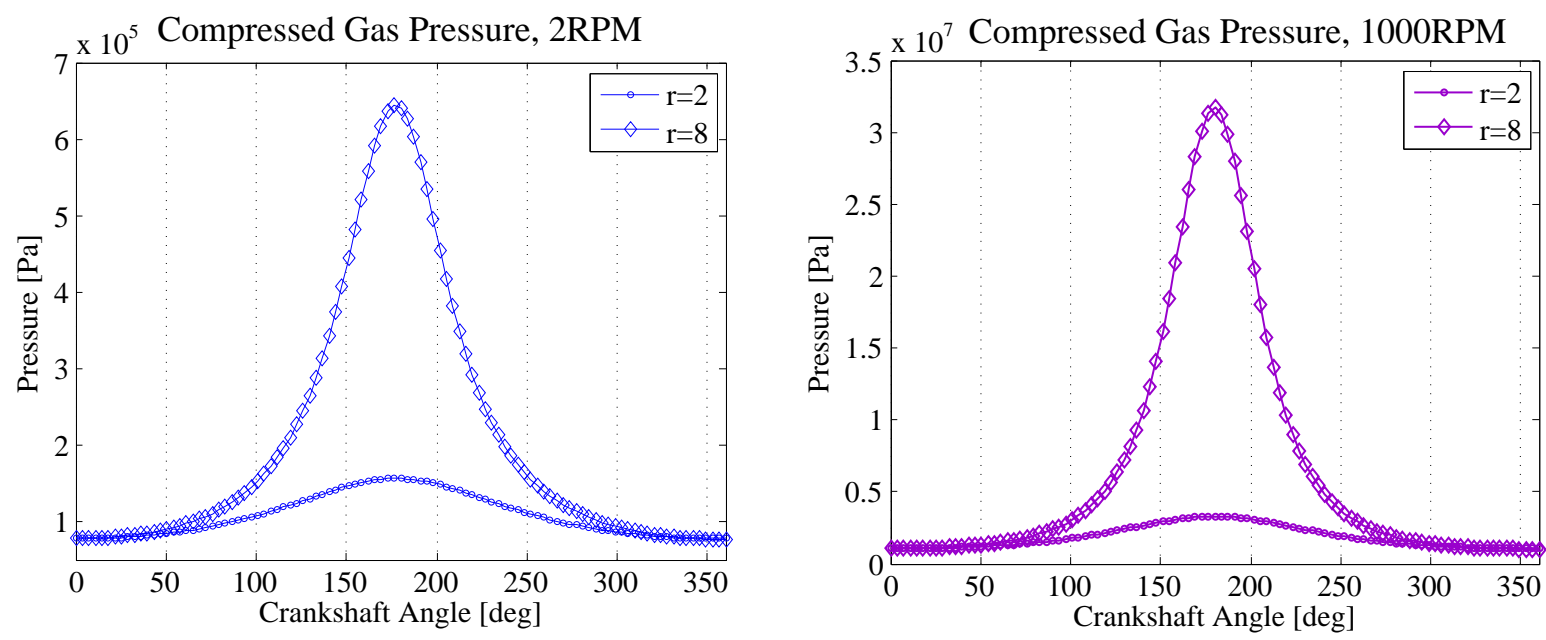

Fig. (8). Simulated pressure for slow and fast runs, $r=2 \& r=8$. 
Density-Averaged Gas Temperature, 2RPM

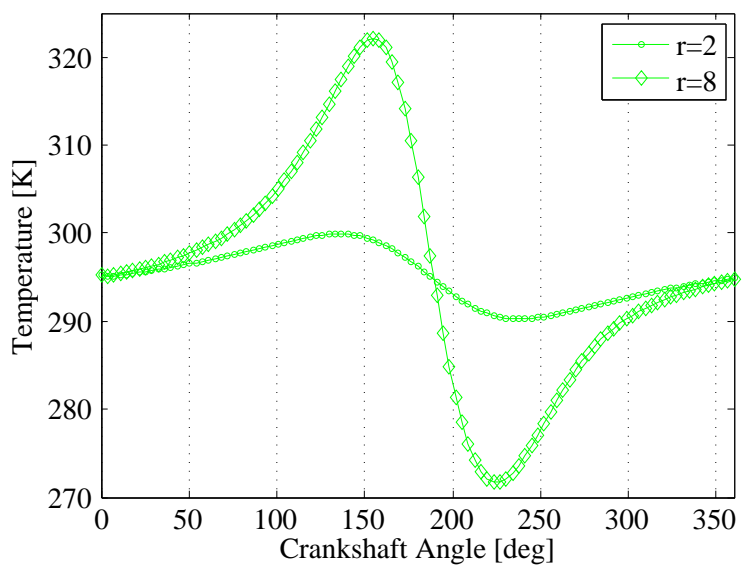

Fig. (9). Simulated temperature for slow and fast runs, $r=2 \& r=8$.

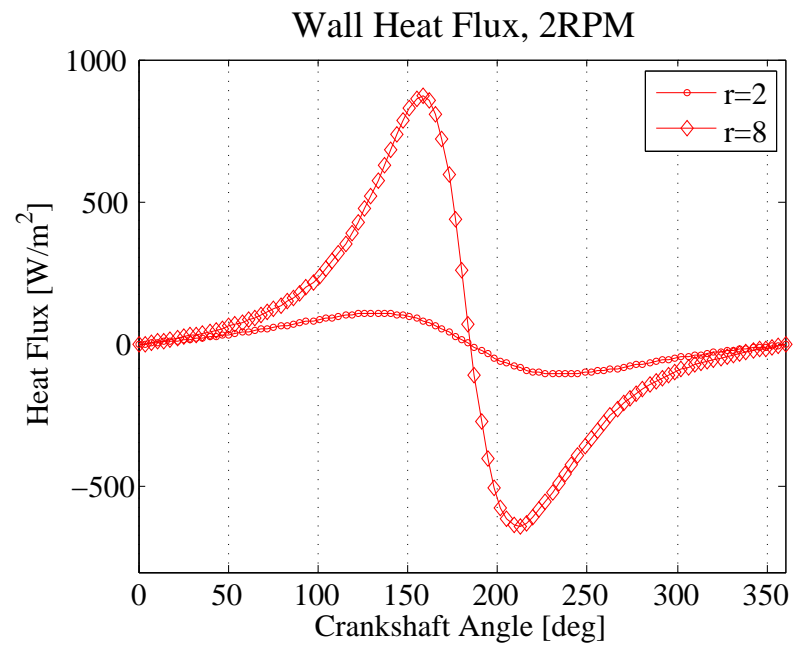

Fig. (10). Simulated wall heat flux for slow and fast runs, $r=2 \& r=8$.

operating gasses, but only helium will be discussed here. Two operating-speed test-cases were 2 and 1000 RPM, as mentioned earlier. Since the experimental results are available only for the compression ratio 2 , in this chapter only this group of simulations will be examined in details and evaluated against measurements.

\section{Experimental Data Processing}

Measured properties in the experimental setup were the absolute compressed gas pressure and the immediate crankshaft angle. Volume was then calculated from the crankshaft angle and driving mechanism geometry. Time signal was provided by an analogue ramp generator. Overall 150 measurements were taken over each cycle. Measurements were recorded once the operating speed and the time-mean cycle pressure became steady, i.e. when the deviation was no more than $0.1 \%$ per cycle (at low speeds this criterion had to be relaxed to $1 \%$ due to the operating speed variations). Heat flux was evaluated as a direct function of pressure, volume and the enclosed mass of the operating fluid, with the use of global thermodynamics (the First Law of Thermodynamics and the ideal-gas equation of state). The algorithm for the heat flux calculation is presented in APPENDIX. Heat-flux
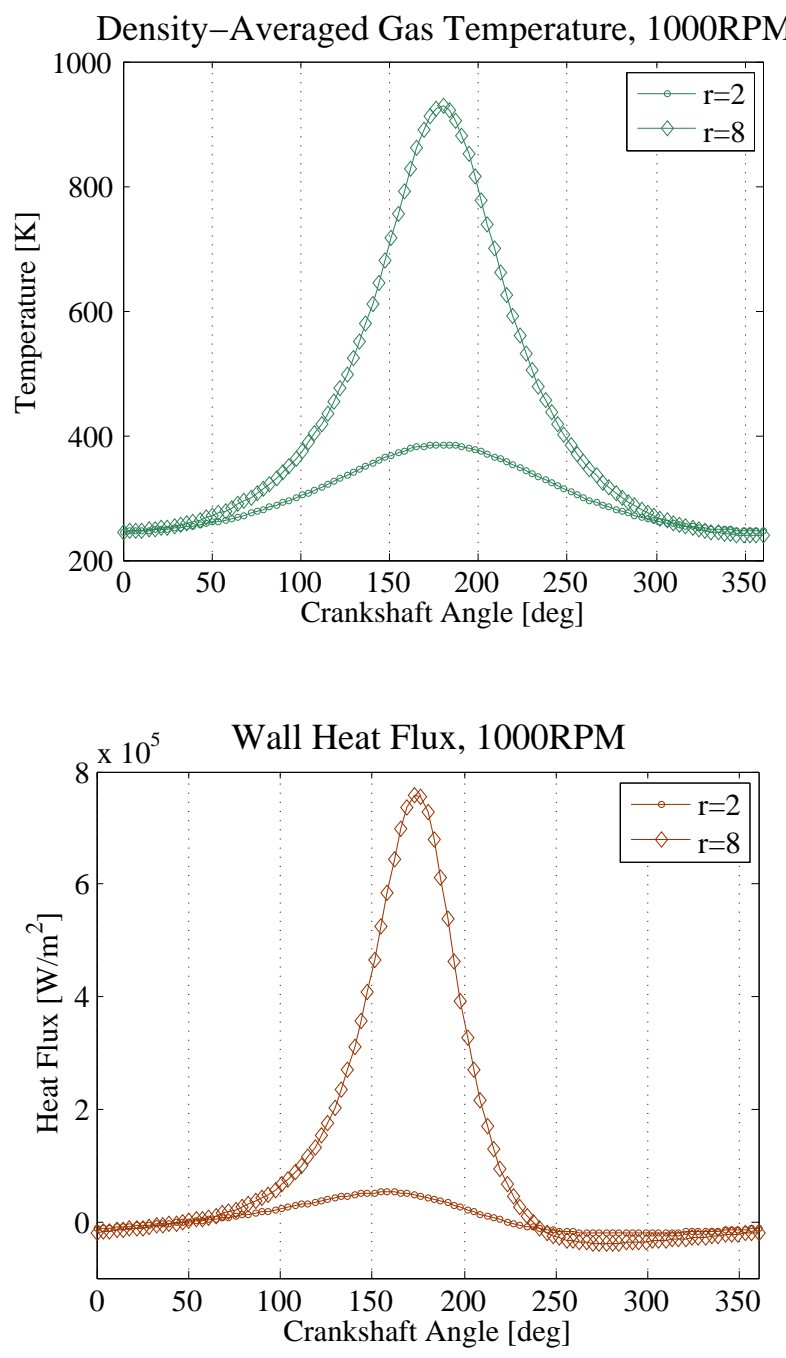

calculated in this way, termed measured, was then compared to the numerically obtained values.

\section{Slow Runs}

As can be seen in Fig. (11), numerically simulated pressure approaches closely the directly measured values. The pressure in the steady state for the slow runs is oscillating periodically between approximately $78 \mathrm{kPa}$ and $160 \mathrm{kPa}$. Simulated pressure is slightly lower at the TDC than the measured pressure and the under-prediction accounts to $2.44 \%$ of the cyclic pressure amplitude. The simulated pressure curve perfectly follows the phase of the measured pressure though, and precedes the adiabatic pressure wave (peak at $180{ }^{\circ} \mathrm{CA}$ ) by approximately $2.5^{\circ} \mathrm{CA}$.

Fig. (12) shows the calculated wall heat flux. It is well predicted at $0-210^{\circ}$ and $300-360{ }^{\circ} \mathrm{CA}$. Heat flux is positive in the compression phase of the cycle, positive being the heat transfer from the gas to the cylinder walls. For the slow runs there is no apparent phase shift between the densitynormalized gas temperature and the heat flux (Fig. 15, discussed later). Past the top-dead-centre, the expansion stroke starts and the heat flux becomes negative, with a negative maximum at approximately $235{ }^{\circ} \mathrm{CA}$. The numerical model under-predicts the reported measured values at this part of 
the cycle; the maximum negative heat flux is underestimated by as much as $50 \%$. Some uncertainty about the measurements arises at this point, as it appears that more heat is being cyclically accumulated than released. That way the gas spring would actually be producing work (nett cyclic heat balance is negative) while the mean compressed-gas temperature is slightly higher than the wall temperature. It is suspected that this discrepancy comes from the deviation from the projected piston trajectory, due to the motor loading at low speeds, and a small phase lag between the pressure and shaft position measurements, as reported by Kornhauser. This would cause errors in calculating the $p d V$ work and correlated calculated heat loss. Nevertheless, it can be concluded that the created ANSYS CFX model can closely predict the transient values of the compressed gas pressure and wall heat flux, for the slowly operating motored gas spring.

\section{Fast Runs}

Boundary conditions in this CFD simulation are identical to those in the slow runs. Initial conditions are set as stated in Table 2; BDC steady-state pressure is $1.05 \mathrm{MPa}$ and temperature $254.5 \mathrm{~K}$.

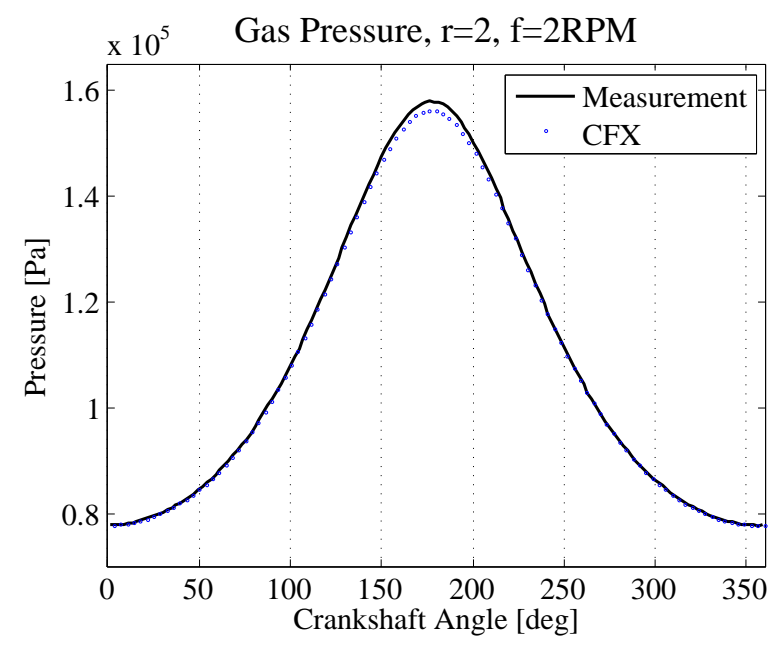

Fig. (11). Measured and simulated gas pressure for the slow run, $r=2$.

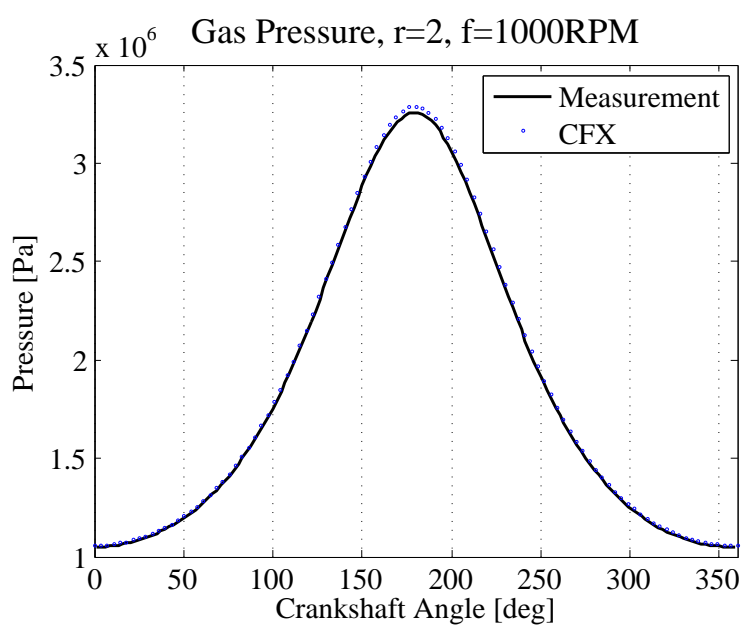

Fig. (13). Measured and simulated gas pressure for the fast run, $r=2$.
Obtained results for gas pressure and wall heat flux are presented in Fig. (13) and Fig. (14). The measured pressure on Fig. (13) is observed to vary between 1 and 3.3MPa, with the maximum closely at the top-dead-centre, $\sim 178^{\circ} \mathrm{CA}$. This is numerically reproduced with an over-prediction of $1.41 \%$ of the pressure amplitude. Wall heat flux is plotted on Fig. (14); the amplitude varies from plus $50 \mathrm{~kW} / \mathrm{m}^{2}$ to -20 $\mathrm{kW} / \mathrm{m}^{2}$, with the peak heat flux in the compression stroke preceding the temperature by approximately $25{ }^{\circ} \mathrm{CA}$. Oscillation in the measured heat flux is suspected to stem from the noise in the measurement pressure signal. Still, the trend of the measurements, and both phasing and amplitude are clearly captured for the higher operating frequency too.

\section{THERMODYNAMIC CONSIDERATIONS}

After validating the numerical models on the set of experimentally obtained data, thermodynamical analyses on the numerical models are made. It is convenient to notice at this point the phasing of the three discussed variables: pressure, temperature and the wall heat flux. These are plotted on Fig. (15), for the slow and fast run, non-dimensionalized and scaled for comparison. For the slow runs, the gas pressure peaks very close to the top-dead-centre (precedes it by ap-

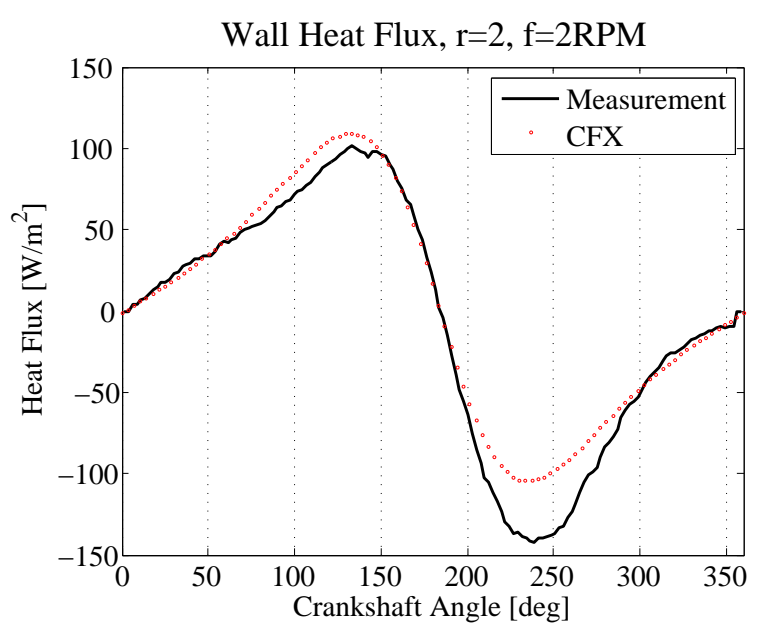

Fig. (12). Measured and simulated wall heat flux for the slow run, $r=2$.

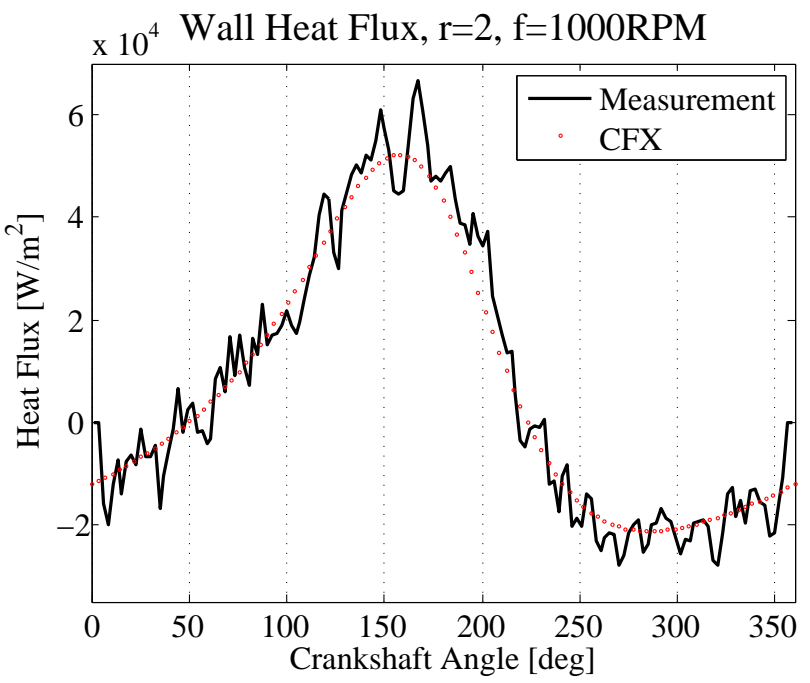

Fig. (14). Measured and simulated wall heat flux for the fast run, $r=2$. 
proximately $2.5^{\circ} \mathrm{CA}$ ), and the gas temperature and wall heat flux are in phase - heat flux follows the heat transfer potential - the temperature difference between the bulk gas and the wall temperature. Heat flux changes from positive to negative close to the $180^{\circ} \mathrm{CA}$ (zero flux occurs at approximately $185^{\circ} \mathrm{CA}$ ), when the piston changes the direction of movement and the gas is basically still, heat flux is proportional to the product of the temperature difference and the gas velocity, as in the Reynolds convection analogy. With the expanding gas, the gas temperature becomes lower than the wall temperature, and with the increasing flow velocity this causes the occurrence of negative heat flux. Negative and positive heat flux peak approximately $50{ }^{\circ} \mathrm{CA}$ after and before TDC, showing strong dependence on the gas temperature, which is also visible from the shape of the two normalized curves shown on the figure. Temperature oscillations are low $(< \pm 5 \mathrm{~K}$, Fig. 9, Table 3) and the slow run thermodynamically approaches the isothermal compression process. This is visible on Fig. (16); it is basically identical in the pressure amplitude, only a small phase shift from the ideal isothermal process can be noticed.

For the fast runs though, the temperature amplitude - wall heat transfer analogy fails. Two effects of the higher operat-

p, T and q phasing, 2RPM

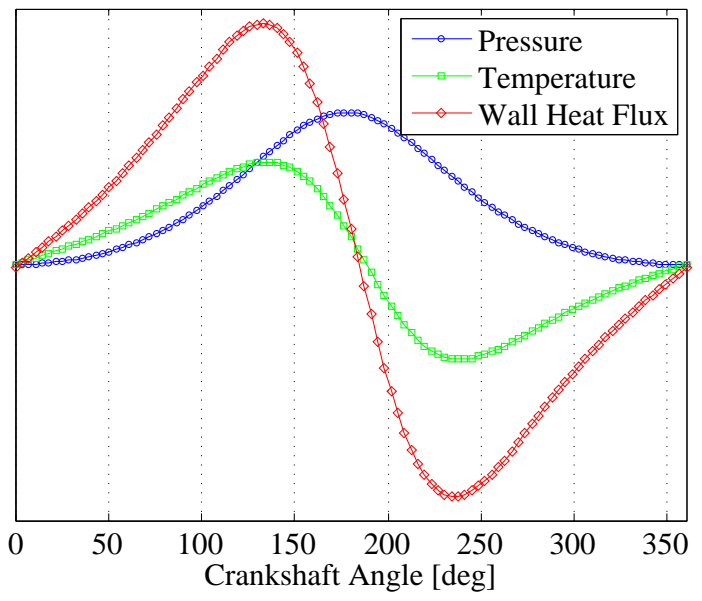

ing frequency are visible: gas temperature oscillation amplitude is much higher than for the slow run, and wall heat flux is out of phase with neither the gas temperature nor the piston speed.

Gas temperature and gas pressure for the fast run are in phase (Fig. (15) right), and peak very close to TDC (preceding it by $2{ }^{\circ} \mathrm{CA}$ ). The dynamics of the compression process here and heat transfer cause the gas temperature not to be as significantly influenced by the wall heat loss as for the slower runs. In the 2 RPM gas spring, compression process is very slow, and the compression work translated to "excess" heat is being instantly transferred to the wall, in that way reducing the internal energy of the gas, and consequently its temperature. Temperature of the gas practically oscillates around the wall level; in the vicinity of the TDC and BDC, when the piston comes to still and changes direction, gas velocities are very low and the gas looses heat by means of conduction to the regions close to the wall and approaches the wall temperature. In the fast run though, much more work is translated into the gas internal energy than taken away by the heat flux. If one takes a look at the approximating equation for the isentropic compression process: $p V^{\kappa}=$ const - for the fast run (Fig. (17)), and the isothermal

\section{p, T and q phasing, 1000RPM}

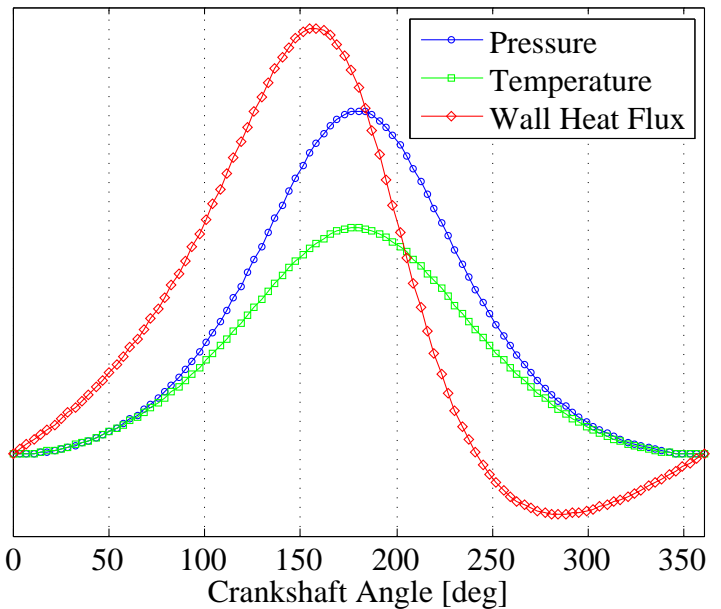

Fig. (15). Phasing of the gas pressure, gas temperature and the wall heat flux for the slow run (left) and the fast run (right).

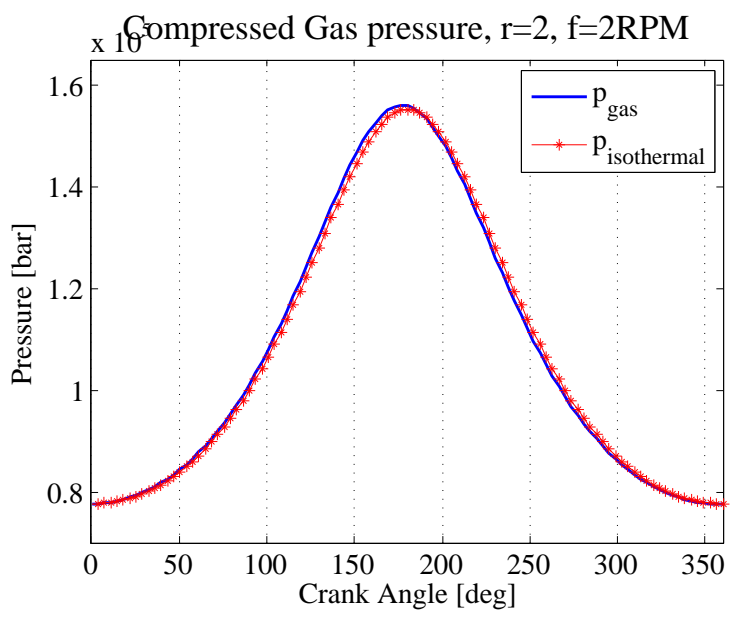

Fig. (16). Approximation of the slow run by the isothermal compression

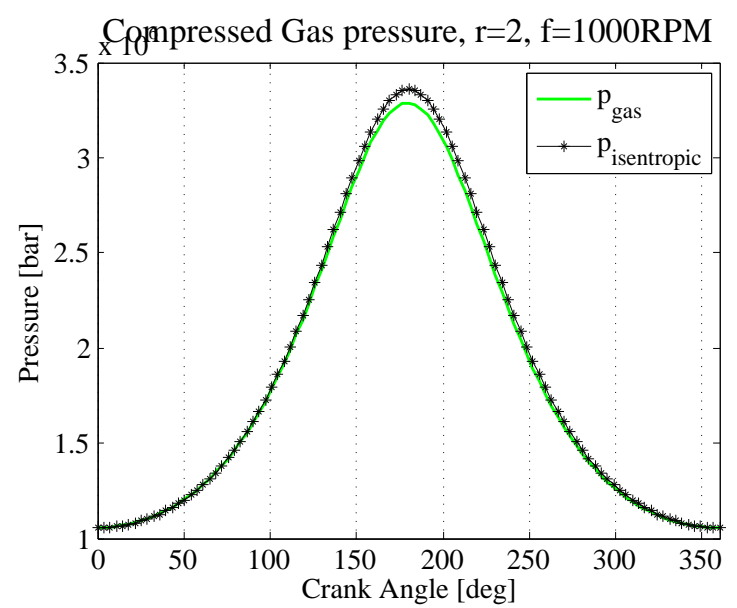

Fig (17). Approximation of the fast run by the isentropic compression 
process: $p V=$ const - for the slow run (Fig. (16)); (where $\kappa$ is the adiabatic compression exponent and $\kappa=1.67$ for helium), it is visible that for the same change in volume $\Delta V$, change in pressure $\Delta p$ will be higher for the higher operating frequencies, since $\kappa>1$. Through the ideal-gas equation of state, $p V=m R T$, this also translates to gas temperatures and confirms the much higher temperature amplitude in the fast run.

Heat flux in the fast runs is shifted "to the right" compared to the slow run; peak heat flux occurs at approximately $155^{\circ} \mathrm{CA}$, thus approximately $65^{\circ} \mathrm{CA}$ later than the maximum piston velocity moment, and $25^{\circ} \mathrm{CA}$ earlier than the maximum temperature difference between the gas and the wall (for the slow run, heat flux was directly proportional to the temperature potential). Zero heat flux also occurs later in the cycle, approximately at $220^{\circ} \mathrm{CA}$. Phenomena related to this will be discussed in the following paragraphs.

On Fig. (18) are plotted the temperature profiles along the half-diameter of the gas spring, as sampled from the computed gas temperatures for nodes along one radius. The range of simulated operating frequencies is extended here, and taken to be 2 to $1500 \mathrm{RPM}$, in order to investigate the development of the temperature profiles in the gas more closely. Temperatures are sampled at the medium axial distance between the TDC and the cylinder top $(38.1 \mathrm{~mm})$, and in the moment of the maximum piston velocities through the cycle $\left(79^{\circ} \mathrm{CA}\right.$ before and after TDC - $101{ }^{\circ} \mathrm{CA}$ and $259^{\circ} \mathrm{CA}$ for compression and expansion stroke). As can be argued from the two figures, with rising operating frequency, distinguished boundary regions next to the wall become more obvious. This boundary region - boundary layers, grows from the Gaussian regular distribution to very narrow close-towall regions. During the fairly slow runs - 2\&10 RPM, wallaffected layer grows to the order of magnitude of the piston diameter; the time scale of the temperature-propagation velocity (heat and mass transfer in the gas) is for the slow run larger than the axial velocity time scale, resulting in the Gaussian-like temperature profile, as met in e.g. developed pipe flow and boundary-layer theory. Temperature of the wall-adjacent gas layers is thus directly correlated to the core temperature, and the heat flux is proportional to the gas-wall temperature difference.

During the high speed runs though, the shear-layer thickness is much smaller than the characteristic length (piston diameter). As the piston speed grows, two regions in the compressed/expanded volume appear: the distinguished viscous boundary layer at the wall, and the well-mixed core. Thermal and velocity boundary layers are formed as the consequence of the finite thermal conductivity of the gas and the discrete value of the molecular viscosity, that with the noslip condition at the wall cause the momentum imposed by the piston displacement to be biased and diffused. Being that there is a time scale related to the propagation of these combined conduction and shear effects, in the fast runs, the turbulent-like core is basically the bulk gas uniformly compressed/expanded and displaced in the axial direction, while the near-wall regions are strongly affected by the presence of the walls. Temperature of the gas at the wall boundaries thus cannot be directly correlated to the bulk gas temperature, and steady-state gas-wall heat transfer analogy fails.

In another view, and stemming from the energy continuity at the gas-wall interface, heat flux at the wall equals the temperature gradient in the gas at the immediate wall boundary, multiplied by the conductivity of the gas (Fourier's law: $\left.q_{x}=-k \cdot d T / d x\right)$. If one looks closely to Fig. (14), it can be notices that the heat flux in the fast gas spring will occur as negative (heat transfer from the walls to the gas) at approximately $220{ }^{\circ} \mathrm{CA}$. On Fig. (19) though, temperature profile along the diameter of the gas spring at this moment is plotted, and it can be seen that the bulk gas temperature is significantly higher than the wall temperature (constant at 295 $\mathrm{K}$ ), while the temperature profile at the wall is horizontal leading to the zero heat flux. This directly displays the inapplicability of the direct correlation assumption to general compression heat transfer. Even for a later expansion moment, visualized on the right figure in Fig. (18) for 1000 RPM, bulk gas temperature is at, or higher than the wall temperature, while the heat flux is already well negative.

\section{Fluid Flow Patterns and Heat Transfer in the Gas Spring}

The occurrence and progression of the flow patterns in the compressing/expanding gas spring is examined on the fast-run simulation and presented on Fig. (20). Frames in the figure represent streamline plots in 6 characteristic instants of the compression cycle, showing half of a symmetry-plane plotted with the lower horizontal line representing the piston, the upper line - the cylinder top, the left vertical boundary is
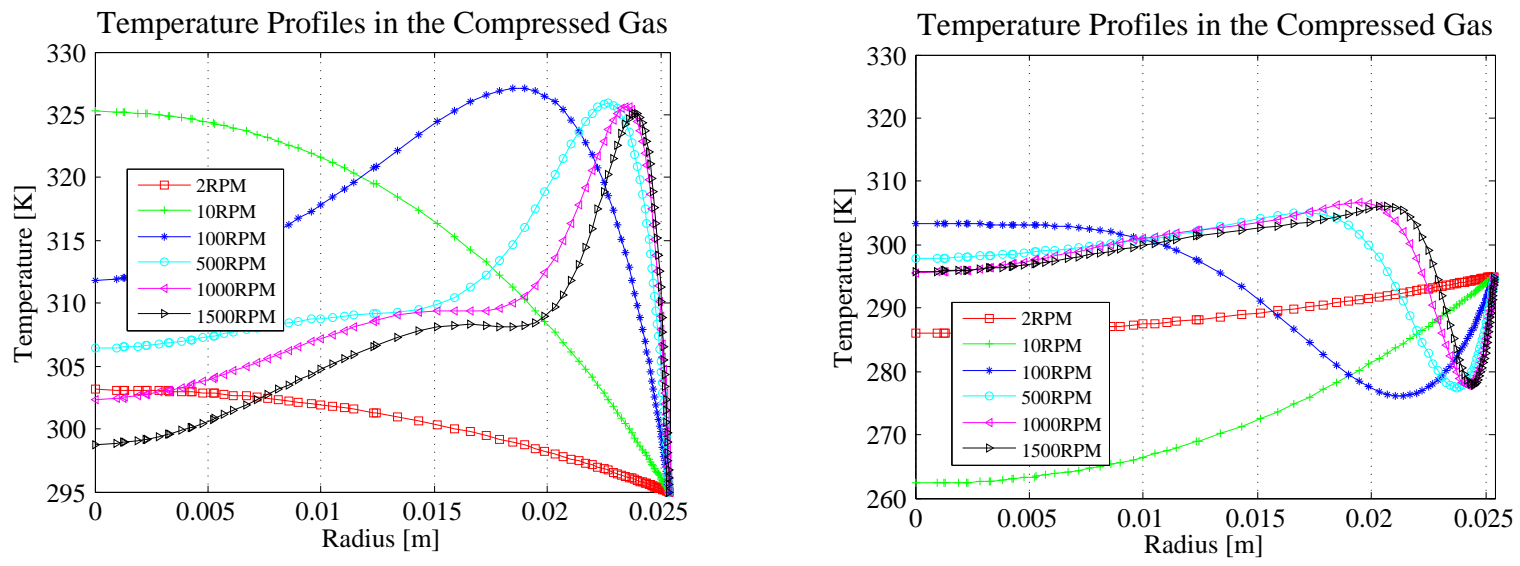

Fig. (18). Temperature profiles in the radial direction, compression stroke (left) and expansion stroke (right). 
the symmetry axis and the right boundary - the cylinder wall. To remind, piston, cylinder top and the cylinder wall are modelled as the solid boundaries, and the no-slip condition is imposed, and piston is the only moving boundary. As the initial condition for the simulation, the captured fluid is at a complete still (all velocities are zero), with uniform properties (temperature, pressure, density) and the piston at BDC position. Displayed is the $10^{\text {th }}$ cycle from the start of simulation, with flows fully developed and the gas spring basically in the steady-state operation. To assist in understanding the complete fluid-flow patterns, the shading on the sample plain in all figures represents the velocity field, with darker tones marking higher velocity regions, according to the accompanying legend.

Temperature Profile in the Gas, 1000RPM, 220degCA

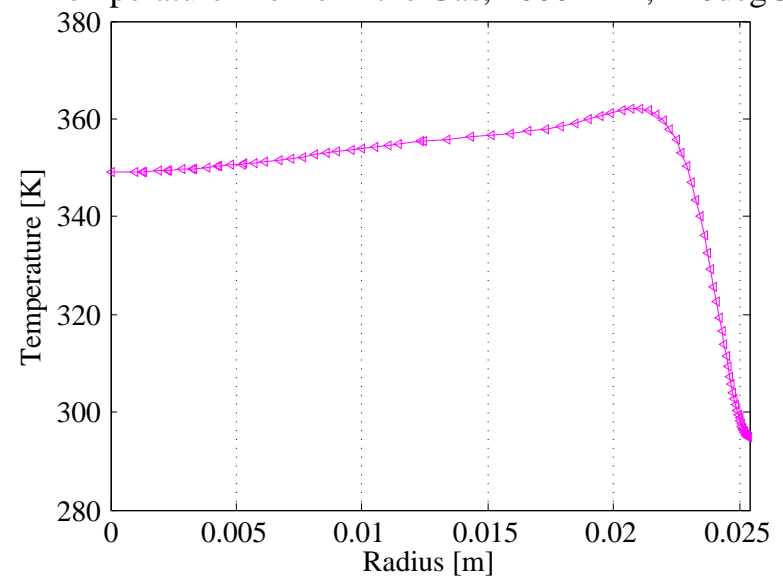

During the compression stroke, notable is the streamlines uniformity during the compression process. Fluid flow follows the naturally dominant axial displacement of the piston, with only some curving near the cylinder head region. Gas velocities are significantly higher close to the piston, with the fluid at the cylinder head appearing to be almost still. In the moment of the change of direction of the piston movement, at the top-dead-centre, fluid viscosity and no-slip condition on the walls "hold" the fluid particles close to the walls, while the rest of the domain is free to flow under the influence of fluid's inertia. With slowing down of the piston and then the change of the mean velocity direction, local vortices are created that gradually lead to three main recirculation areas (Fig. 20). One is on the cylinder wall bound-

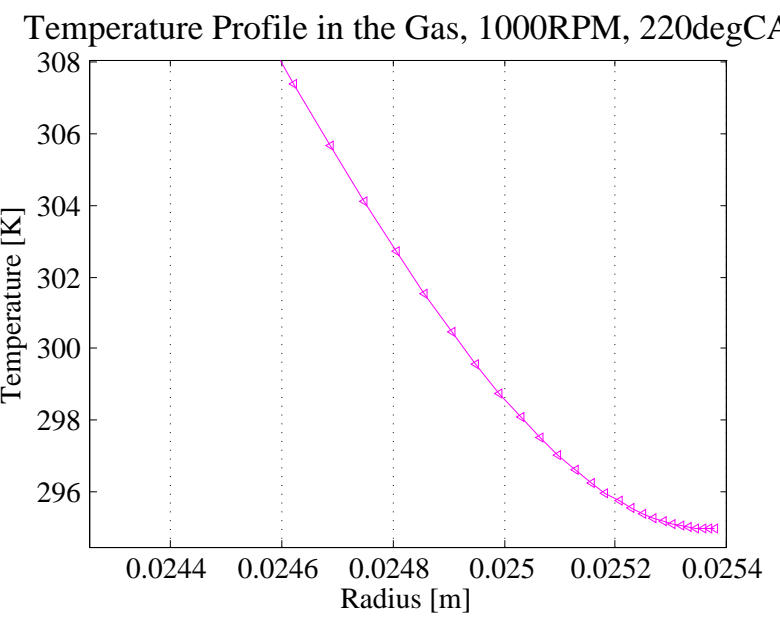

Fig. (19). Gas temperature radial profile at $220^{\circ} \mathrm{CA}$ along the radius and enlarged at the wall.

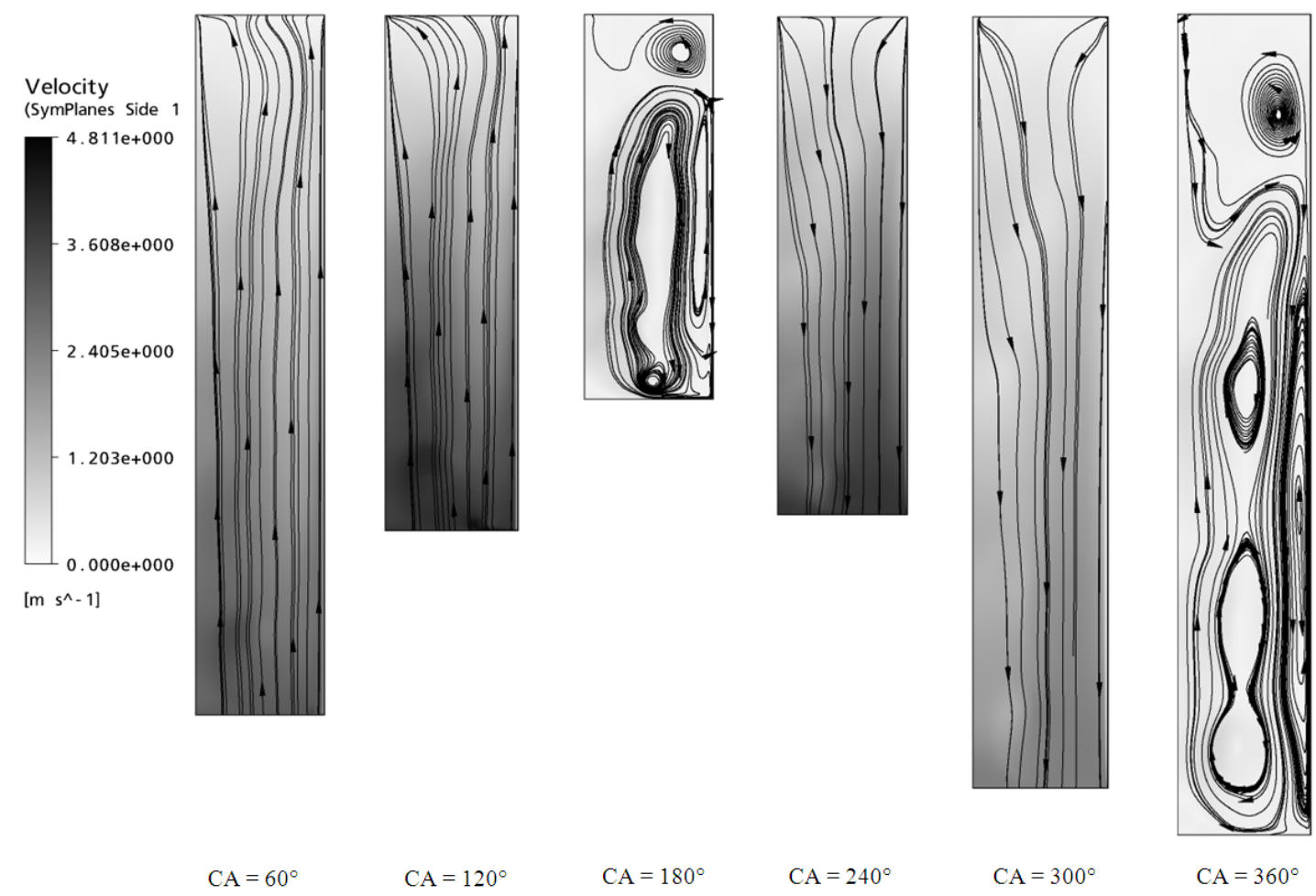

Fig. (20). Velocity field and streamlines during one cycle. 
ary, one is in the "dead" corner of cylinder and head, and the third is in the bulk gas area. It should be noticed, though, that the velocities of the fluid particles are very low in this part of the cycle. With expansion and piston moving in the other direction, flow again takes the nearly uniform vertical order, up to the BDC moment, when vorticing and recirculation areas appear again.

Flow patterns mentioned above govern the heat transfer process during the compression/expansion cycle. During the upward and downward strokes, while the piston is moving with a significant velocity, flow inside the gas spring is very stratified, and the dominating heat transfer mechanism in the fluid is conduction. There is no gas inflow or domain geometry to increase the mixing or stimulate the radial velocity component (the vorticing is caused only by viscosity-driven shearing effects in layers close to the wall), and the redistribution of gas temperature in the radial direction is very limited. With the low thermal conductivity of the gas, for the fast run conduction is a much slower process than the instant warming-up and cooling of the axial layers in the gas, imposed by the piston movement and compression. This causes the specific temperature profiles shown typically in Fig. (18). Fluid in the vicinity of the surrounding walls is affected by the thermal mass of the solid and forms thermal boundary layers. These boundary layers can carry a significant part of the volume-enclosed gas mass (up to 30-40\%, [9]) due to high density gradients and act as buffers - high density insulating layers that actively interact in the energy exchange with the core only in the moments of changing piston motion direction, i.e. at TDC and BDC. The flow structure is then disturbed and the warm/cold core gas recirculates to the wall, only then forcing the actual convective heat transfer. In this way the instantaneous wall heat flux is not directly dependent on the temperature of the gas-spring core, but rather on the dynamics of the compression process, extent of mixing of the fluid flows and the interaction between the core and the wall, through the bounding gas layers.

Fig. (21) displays the forming and disappearance of the vortices around the TDC in more details. On Fig. (21), the interaction of the wall-affected layers and the shearing adjacent streams is visible. The first vortices are formed in the
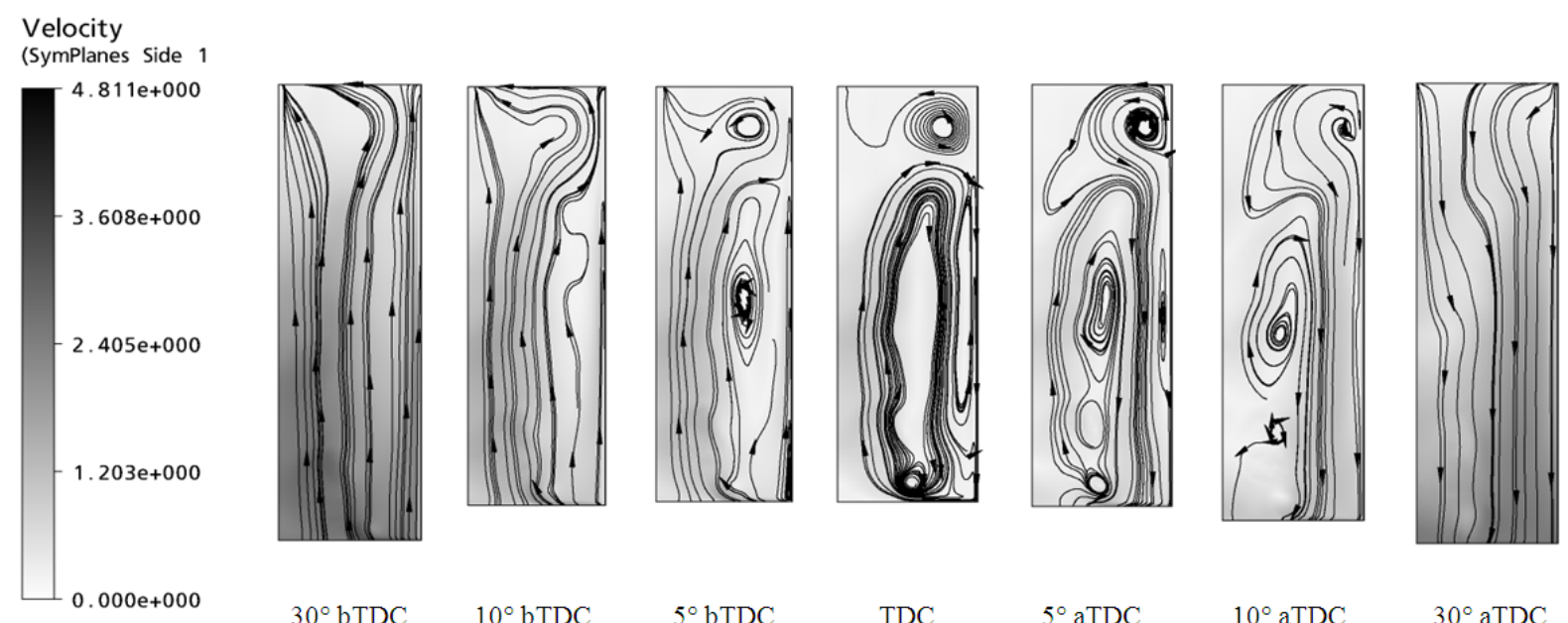

$\left[\mathrm{m} \mathrm{s}^{\wedge}-1\right]$ vicinity of the vertical wall and the corner of the cylinder wall and head. Second vortex is formed at the very wall boundary due to the no-velocity condition; with the large central vortex rotating in the clock-wise direction, sheering forces direct the new swirl in the counter-clockwise direction and the core fluid is brought to the wall. With expansion, vortex at the wall disperses first and the central vortex is moved inwards. The latest swirl disappears; the vortices decay and the streamlines return to vertically structured order. [3] and [8] in their work publish considerations of turbulence occurring inside the gas spring for the fast run. Turbulence parameters can be examined at this point to investigate for the actual presence of developed turbulence. Eddy-viscosity and the geometry Reynolds number can be taken as critical properties [5]. Eddy viscosity, as obtained from the computations is in the order $10^{3}$ smaller than the molecular $\left(\mu_{t, \text { max }} \approx 1 \mathrm{e}^{-8}, \mu=1.86 \mathrm{e}^{-5}\right)$; in the developed turbulent pipe flows it is three orders higher than the dynamic viscosity [5]. At the same time, Reynolds number estimated on basis of the piston speed, cylinder diameter and cyclemean kinematic viscosity peaks at $\sim 23000$. There are no published studies on critical Reynolds numbers in this type of flow geometries, but it is known that for pipe flows established turbulence criterion is $R e>10^{4}$. In that regard, even though vorticing regions are present next to structured layers, this happens only in the low velocity parts of the cycle, thus with gas Reynolds numbers far below the established turbulence limits or approaching zero. In the same view, in the moments of the maximum $R e$, no swirling is visible in the compressed flow. It is discussible if the flows occurring in the presented gas spring can be regarded to as turbulent as defined in the established fluid-mechanics terminology; even if transition to turbulent regime starts in the highest piston speed moment, this is very quickly biased by slowing down of the piston and changing of the flow direction.

\section{CONCLUSIONS}

Direct correlations between the measurable gas-wall temperature difference and instantaneous wall heat transfer are possible only for developed, steady state flows; typical examples being the steady in-pipe flow, flow around the cyl-

Fig. (21). Development of the flow patterns close to TDC. 
inder or over a plate. These correlations fail for closed reciprocating piston-cylinder geometries. To date there are no analytical models to quantitatively, or qualitatively predict the instantaneous wall heat flux. Numerical models are developed and presented here with the use of the finitevolume numerical package ANSYS CFX11.0, to simulate the occurring thermodynamic phenomena.

Characteristic properties such as the compressed gas pressure and heat transfer from the gas to the surrounding solid are examined. For typically slow runs, wall heat flux is in phase and proportional to the gas-wall temperature difference. For the typically fast runs, gas temperature and pressure are in phase, while the peak heat transfer precedes the maximum gas-wall temperature difference by $25^{\circ} \mathrm{CA}$ and occurs approximately $65^{\circ} \mathrm{CA}$ later than the maximum piston velocity moment. Simulated results are compared to the experimental database obtained at MIT. Numerical models very successfully predict the measured values, with very little error introduced and prove to be a powerful tool for CFD design.

In view of the relevance to the convective heat transfer, fluid-flow patterns occurring in the gas springs are analysed. Timescale of propagation and the shape of thermal boundary layers are discussed. During the compression and expansion strokes flow inside the gas spring is very stratified, and the dominating heat transfer mechanism is conduction. The flow structure is only disturbed at the dead centre positions of the piston, when the core gas recirculates to the wall and forces the actual convective heat transfer. In this way the instantaneous wall heat flux is not directly dependent on the temperature of the gas-spring core, but rather on the dynamics of the compression process and existence of the boundary layers in the compressed gas.

\section{APPENDIX - HEAT FLUX CALCULATION}

Heat flux evaluation procedure by Kornhauser [3] is presented by the following set of equations. Heat transfer rate was calculated from the pressure, volume and time data together with the gas equation of state and the first law of thermodynamics. The time derivatives were evaluated applying a five-point least squares fit model [10] to the timedependent measured data. Since data was collected on a crank-angle basis rather than time-basis, and the angular frequency varied slightly over the cycle, data was interpolated to a time basis before differentiation and interpolated back to a crank-angle basis afterwards.

From the initial values of pressure, temperature and volume of the gas, gas temperature at every instant of the cycle is calculated as:

$T_{i}=\frac{p_{i} V_{i}}{\frac{p_{\text {init }} V_{\text {init }}}{T_{\text {init }}}}$,

for a constant mass of the compressed gas.

Time-derivatives of the temperature and volume are:

$\left(\frac{d T}{d t}\right)_{i}=\frac{5 \sum_{j=i-2}^{i+2} T_{j} t_{j}-\sum_{j=i-2}^{i+2} T_{j} \sum_{j+i-2}^{i+2} t_{j}}{5 \sum_{j=i-2}^{i+2} t_{j}^{2}-\left(\sum_{j=i-2}^{i+2} t_{j}\right)^{2}}$ and $\left(\frac{d V}{d t}\right)_{i}=\frac{\mathbf{5} \sum_{j=i-2}^{i+2} V_{f} t_{j}-\sum_{j=i-2}^{i+2} V_{j} \sum_{j=i-2}^{i+2} t_{j}}{\mathbf{5} \sum_{j=i-2}^{i+2} t_{j}^{2}-\left(\sum_{j=i-2}^{i+2} t_{j}\right)^{2}}$

The rate of change of internal energy was then

$\left(\frac{d U}{d t}\right)_{i}=m c_{v}\left(\frac{d T}{d t}\right)_{i}$

and the rate of heat transfer, with both work and heat defined as positive out of the system:

$Q_{\mathrm{t}}=-\left[m c_{v}\left(\frac{d T}{d t}\right)_{i}+p_{i}\left(\frac{d V}{d t}\right)_{i}\right]$.

Wall heat flux at a time instant was then:

$\dot{q}_{\mathrm{t}}=\frac{Q_{t}}{A}$,

with $A$ being the instantaneous heat-transfer surface.

\section{ACKNOWLEDGMENTS}

This project is sponsored by Technologiestichting STW Netherlands. Many thanks also to Prof. Kornhauser presently at Virginia State University for making his measurement data available.

\section{NOMENCLATURE}

$c$

$D$

$f$

$k$

$l$

$m$

$p$

PistDisp $=$ Instant piston displacement

$r$

$R$

$\dot{q}$

$\dot{Q}$
$=$ Crankshaft throw

$=$ Heat transfer area

$=$ Constant volume specific heat capacity

$=$ Cylinder diameter

$=$ Operating frequency

$=$ Gas conductivity

$=$ Connecting rod length

$=\quad$ Mass of the compressed gas

$=$ Gas pressure

$=$ Pressure compression ratio

$=\quad$ Specific gas constant

$=$ Heat flux

$=$ Heat transfer rate

$=$ Piston stroke

$=$ Gas temperature

$=$ Time

$=$ Turbulent, maximal cyclic value

$=\quad$ Gas internal energy

$=$ Gas volume

$=$ Adiabatic exponent

$=$ Dynamic gas viscosity

$=$ Crank angle

$=$ Degrees crank-angle 


\section{Subscripts}

$\begin{array}{lll}i, j & = & \text { Integers } \\ \text { init } & = & \text { Initial }\end{array}$

\section{REFERENCES}

[1] W.J.D. Annand and D. Pinfold, "Heat Transfer in the Cylinder of a Motored Reciprocating Engine," Society of Automotive Engineers, SAE Paper 800457, 1980.

[2] B Lawton, "Effect of compression and expansion on instantaneous heat transfer in reciprocating internal combustion engines," MIMechE Paper, vol. 201, no. A3, 1987.

[3] A.A. Kornhauser, "Gas-Wall Heat Transfer During Compression and Expansion," Massachusetts Institute of Technology, $\mathrm{PhD}$ Thesis 1989.
[4] K.P. Lee, "A Simplistic Model of Cyclic Heat Transfer Phenomena in Closed Spaces," in 18th IECEC, 1983, pp. 720-730.

[5] ANSYS Inc, ANSYS CFX Release 10.0: Instalation and Overview Notes, 2005.

[6] U. Lekić and J.B.W Kok, "Heat Flows in Piston Compressors," in 5th EUROTHERM, 2008, FCV_4.

[7] R.P. Adair, E.B. Qvale, J.P. Pearson, "Instantaneous heat transfer to the cylinder wall in reciprocating compressors," in 1972 Purdue Compressor Technology Conference, USA, 1972.

[8] H.G. Jak, "Heat Transfer in a Gas Spring," University of Twente, M.Sc.Thesis 2001.

[9] E.J. Heywood and J.B. Lyford-Pike, "Thermal boundary layer thickness in the cylinder pf a spark-ignition engine," International Journal of Heat and Mass Transfer, vol. 27, pp. 1873-1878, 1984.

[10] D.H. Menzel, Fundamental Formulas of Physics, England: Dover, 1960, vol. 1.

(C) Lekić and Kok; Licensee Bentham Open.

This is an open access article licensed under the terms of the Creative Commons Attribution Non-Commercial License (http://creativecommons.org/licenses/by-nc/3.0/) which permits unrestricted, non-commercial use, distribution and reproduction in any medium, provided the work is properly cited. 